\title{
Comparison of antimicrobial effect of various oils mixed with zinc oxide - an ex vivo, in vitro study
}

\author{
Faizal C. Peedikayil, Akhila Ansari, Chandru TP, Soni Kottayi, Aparna TP, \\ Shabnam Ismail \\ Department of Pediatric Dentistry, Kannur Dental College, Kannur, Kerala, India
}

\begin{abstract}
Background. Success of endodontic treatment depends on complete elimination and various factors such as the antimicrobial activity of the obturating materials. Therefore a study was conducted to find the most prevalent species in the infected canals of primary teeth and to compare the antimicrobial effect of zinc oxide with various oils against most prevalent root canal pathogens.

Materials and method. The study was conducted on 100 children in the age group of 3 to 12 years with infected root canal in primary teeth. The sample was subjected to various microbiological analysis to identify the colonies. Following identification, the most common organism was taken and antimicrobial activity of zinc oxide eugenol, zinc oxide with coconut oil, zinc oxide with peppermint oil, zinc oxide with cinnamon oil, zinc oxide with tea tree oil on plates were tested. The zone of inhibition was measured and datas were tested for statistical significance.

Results. Enterococcus faecalis was the most prevalent organism. It was seen that zinc oxide with tea tree oil had shown maximum antimicrobial activity against Enterococcus faecalis followed by zinc oxide with coconut oil, zinc oxide with peppermint oil, zinc oxide with cinnamon oil and zinc oxide with eugenol. Conclusions. The study shows that Enterococcus faecalis was the most prevalent microbe in the infected root canals of primary molars. Zinc oxide with oil mixtures also has shown significant antimicrobial activity against Enterococcus faecalis when compared to zinc oxide eugenol.
\end{abstract}

Keywords: antimicrobial activity, zinc oxide, Enterococcus faecalis, Streptococcus mutans, essential oils

\section{INTRODUCTION}

Oral cavity is a natural and favourable habitat for about 700 different types of organisms due to the presence of nutrients, epithelial debris and secretions. The imbalance due to oral environmental factors leads to changes in concentration of oral microbiomes and lead to diseases such as dental caries (1). These bacteria invades through diseased tooth structures to the root and periapical tissue and has been implicated in the infections of endodontic origin (2).

The endodontic infection in deciduous teeth with pulp necrosis is of polymicrobial nature with predominance of anaerobic bacteria. However, these mi- croorganisms disseminated in root canal system may persist even after cleaning and shaping of the root. The use of root canal filling materials with antibacterial properties should be able to eliminate residual pathogens, neutralize their toxic products and prevent canal reinfection in order to create a favourable environment for the healing process to proceed in periapical region $(3,4)$. It is therefore imperative that the endodontic filling materials used in primary teeth possess certain amount of antimicrobial activity.

Traditionally zinc oxide eugenol has long been used as an endodontic obturating material in primary tooth. Zinc oxide eugenol has been shown to cause slow resorption, irritation to oral tissues in children, 
periapical tissue irritation, bone and cemental necrosis, affects the path of eruption of succedaneous tooth and has only limited antimicrobial action (5). Therefore few researchers does not consider zinc oxide eugenol as an ideal root canal filling material (6). So in search of a liquid that can replace eugenol, this study was undertaken to compare the antimicrobial action of zinc oxide with other oils such as coconut oil, cinnamon oil, peppermint oil, tea tree oil against the common root canal pathogen. Many natural oils like coconut oil, cinnamon oil, peppermint oil, tea tree oil that have been used for treatment of various medical and dental problems since ancient times. They possess antibacterial, antifungal, and antioxidant properties $(7,8)$.

Therefore this study was under taken to find the predominant organisms in infected root canals and also to find the action of various formulation of zinc oxide with coconut oil, tea tree oil, peppermint oil and cinnamon oil against the most prevalent pathogenic organisms.

\section{MATERIALS AND METHODS}

This ex vivo-in vitro study was done in the Department of Paediatric Dentistry of a Dental College in association with Department of Microbiology of a Medical College. Before the start of the study institutional ethical committee approval (KDC/ETH/18/ PED11/1) as taken.

The study consisted of two parts:

Step 1 - To identify most common organisms associated with infected primary root canal.

Step 2 - To assess the antimicrobial effect of zinc oxide with eugenol, zinc oxide with coconut oil, zinc oxide with peppermint oil, zinc oxide with cinnamon oil, and zinc oxide with tea tree oil against most common pathogens.

The study population comprised of 100 children aged between 3 to 12 years, having irreversible pulpal infections or infected root canals. The sample size was calculated using the formula $=\mathrm{Z} 1 \alpha / 22 \mathrm{SD} 2 \mathrm{~d} 2$ $\mathrm{Z} 1 \alpha / 2=1.96$ at $95 \%$ confidence interval. $\mathrm{SD}=$ standard deviation of variable. Value of standard deviation can be taken from previously done study. $\mathrm{d}=$ absolute error. Therefore the sample size $=1.962(25) 2=96$, rounded to 100 for study purpose. The inclusion criteria was children between 3 and 12 years with irreversible pulpal infection and infected root canal of primary lower molars. The exclusion criteria for the study was children with oral candidiasis, children with any congenital or systemic or contagious diseases and children on antibiotics. The patient's parent /guardian was explained about the study and written informed consent was taken before the onset of the study.

After access opening, microbial samples were collected using a sterile paper point by passing it through the infected root canal of the primary molar for 30 seconds. Following removal from the canal, the paper point was immediately placed in cryovials and was transferred to microbiology department for analysis of various root canal pathogens. Lab procedures were conducted according to CLSI (Clinical and Laboratory Standard Institute) guidelines. The sample were inoculated on Blood Agar and Mac Conkey Agar plates and incubated at $37^{\circ} \mathrm{C}$ for $24 \mathrm{hrs}$. Laboratory procedures such as Gram staining and tests such as catalase test, salt tolerance test, oxidase test were done. Enterococcus group was confirmed through bile esculin test. Other colonies identified were confirmed through the Vitek test.

In the second part of the study, a standard inoculum was obtained by passing single colony of most prevalent microorganism in nutrient broth and the turbidity of broth was adjusted with McFarland 0.5 standard. By using lawn technique, microbial colonies were spread uniformly on Muller Hinton agar media. Then 6 wells each $4 \mathrm{~mm}$ in depth and $6 \mathrm{~mm}$ in diameter were made in each of the agar plates with equal distance from each other. To each well zinc oxide with peppermint oil (Group 1), zinc oxide with tea tree oil (Group 2), zinc oxide with cinnamon oil (Group 3), zinc oxide with coconut oil (Group 4), zinc oxide with eugenol (Group 5) were placed. All the plates were kept in incubator at $37^{\circ} \mathrm{C}$ for $24 \mathrm{hrs}$ and the diameter of zones of inhibition was measured and recorded.

\section{RESULTS}

The specimens from 100 patients in the age group of 3 to 12 years were taken for this study The patients constituted $62 \%$ females and $48 \%$ males.

Of the microorganisms isolated 55\% were Enterococcus faecalis which is a Gram-positive facultative anaerobe, $30 \%$ were Streptococcus mutans which is a facultatively anaerobe, $5 \%$ were Peptostreptococcus which is an anaerobic, Gram-positive, non-spore forming bacteria, 6\% were Porphyromonas gingi- 
valis which is a black-pigmented, anaerobic, nonmotile Gram-negative species, $4 \%$ were Actinomyces species which gram-positive facultative anaerobe (Table 1).

TABLE 1. Prevalence of microorganisms from root canals of decidous molar

\begin{tabular}{|l|c|}
\hline Species & Prevalence \\
\hline Enterococcus faecalis & $55 \%$ \\
Streptococcus mutans & $30 \%$ \\
Peptostreptococcus & $5 \%$ \\
Porphyromonas gingivalis & $6 \%$ \\
Actinomyces & $4 \%$ \\
\hline
\end{tabular}

Figure 1 shows zone of inhibition on Enterococcus faecalis. Table 2 shows mean zone of inhibition values of different groups against Enterococcus faecalis. Diameters of zones of inhibition in $\mathrm{mm}$ for zinc oxide with tea tree oil paste were largest i.e.; $41.1667 \pm 2.40$ followed by zinc oxide with coconut oil with a zone of inhibition of $40.333 \pm 1.50$ and zinc oxide with peppermint oil with a zone of inhibition of $37.6667 \pm 2.06$ followed by zinc oxide with cinnamon oil with a zone of inhibition of $36.5000 \pm 2.07$ and zinc oxide with eugenol paste was least with a zone of inhibition of $29.0000 \pm 1.09$.

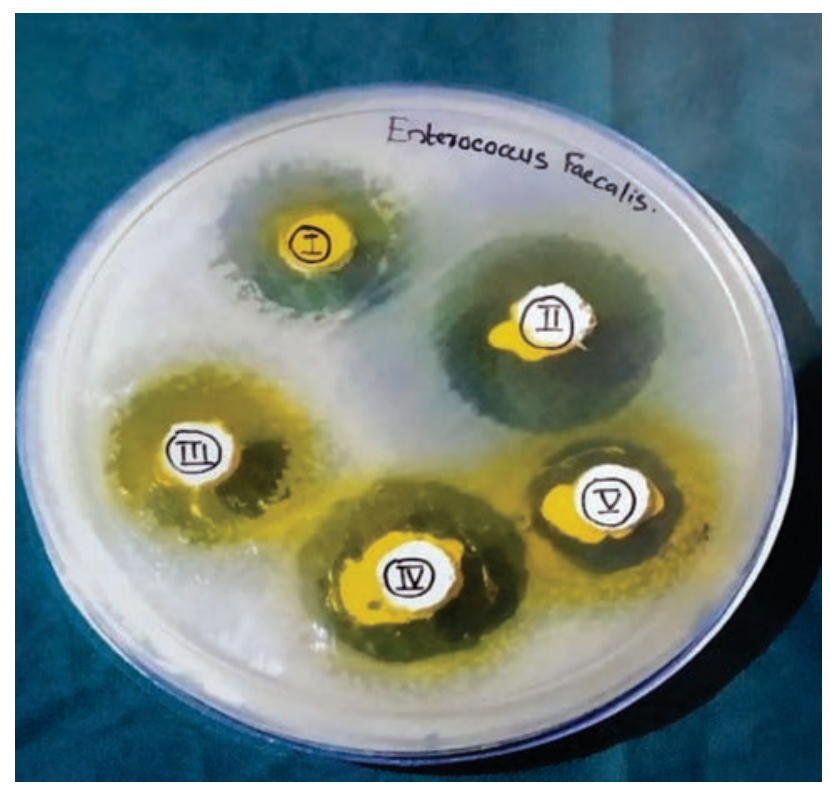

FIGURE 1. Zone of inhibition of various oils mixed with zinc oxide powder against Enterococcus faecalis

TABLE 2. Mean zone of inhibition values of different groups against Enterococcus faecalis

\begin{tabular}{|l|l|}
\hline Groups & Mean \pm SD \\
\hline Group 1 & $37.6667 \pm 2.06$ \\
\hline Group 2 & $41.1667 \pm 2.40$ \\
\hline Group 3 & $36.5000 \pm 2.07$ \\
\hline Group 4 & $40.3333 \pm 1.50$ \\
\hline Group 5 & $29.0000 \pm 1.09$ \\
\hline
\end{tabular}

Table 3 shows the antimicrobial effect using the One way ANOVA. The difference between and within the group was found to be highly statistically significant $(p=0.00)$

TABLE 3. Antimicrobial effect of $Z n O P, Z n o t, Z n O$ $\mathrm{Ci}, \mathrm{ZnO} \mathrm{Co}, \mathrm{Zno} E$ in the form of zone of inhibition on Enterococcus faecalis using One Way ANOVA

\begin{tabular}{|l|c|c|c|c|c|}
\hline & $\begin{array}{c}\text { Sum of } \\
\text { Squares }\end{array}$ & df & $\begin{array}{c}\text { Mean } \\
\text { Square }\end{array}$ & F & Sig. \\
\hline Between groups & 558.867 & 4 & 139.717 & 39.246 & .000 \\
\hline Within groups & 89.000 & 25 & 3.560 & & \\
\hline Total & 647.867 & 29 & & & \\
\hline
\end{tabular}

Table 4 shows the antimicrobial effect of zinc oxide with peppermint oil, zinc oxide with tea tree oil, zinc oxide with coconut oil, zinc oxide with cinnamon oil, zinc oxide with eugenol showing zone of inhibition on Enterococcus faecalis using Multiple Comparison Tukey's test. When antimicrobial effect of zinc oxide with peppermint oil was compared with zinc oxide with eugenol and zinc oxide with tea tree oil, it was found to be highly statistically significant $(\mathrm{p}<0.05)$. When zinc oxide with tea tree oil was compared with zinc oxide with cinnamon and zinc oxide with eugenol, the difference was found to be highly significant $(\mathrm{p}$ value $=.000$ ). The antimicrobial effect of zinc oxide with cinnamon was found to to be statistically significant when compared with zinc oxide with coconut oil, zinc oxide with eugenol ( $\mathrm{p}<$ 0.05). Zinc oxide with coconut oil was found to be statistically significant when compared with zinc oxide with cinnamon and zinc oxide with eugenol $(\mathrm{p}=$ 0.05 ). No statistical difference was found when zinc oxide with peppermint oil was compared with zinc oxide with cinnamon and zinc oxide with coconut oil.

\section{DISCUSSION}

In the present study, teeth with infected root canal were selected. Microbial samples were collected using paper points placed in canals. Grossman et al. supported the use of sterile paper points for obtaining the root canal samples and showed that 1-10 microorganisms are optimally needed for obtaining growth through culture procedures (9).

The results of our study shows the most common species isolated from infected root canals were Enterococcus faecalis. Colonies of Streptococcus mutans. peptostreptococcus, porphyromonas gingivalis and actinomyces species were also observed in the study 
TABLE 4. Antimicrobial effect of ZnO P, Zno T, ZnO Ci, ZnO Co, Zno E in the form of zone of inhibition on Enterococcus faecalis using Multiple Comparison Tukey's Test

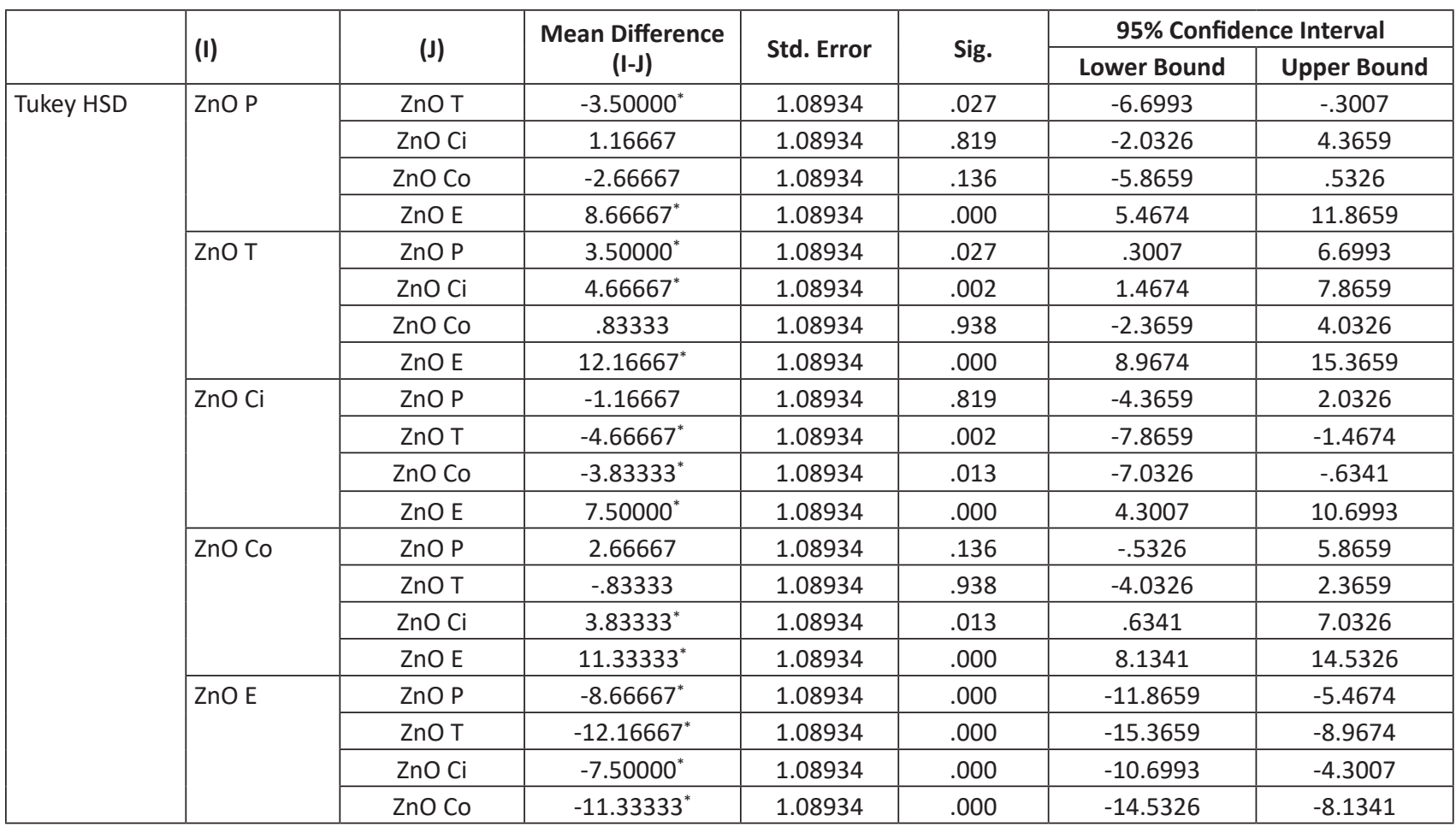

which confirms the polymicrobial nature of colonization of infected root canals of deciduous molars. The microorganisms of the root canal has several characteristics leading biologic and pathogenic events such as antigenicity, mitogenic characteristics, chemotaxis, histolysis with enzymes and activation of host cells (10).

Various studies have analysed the composition of microorganisms in the root canal with a persistent apical lesion. The analysis of these studies showed varied predominance of different species (11). Pinheiro et al. found in their study that, Enterococcus faecalis was the most frequently recovered bacterial species among the facultative anaerobic species and gram positive microorganisms (12).

Enterococcus faecalis is occasionally found in cases of primary endodontic infections as well as in failed endodontic infections (13). Enterococci survive in root canal infections where nutrients are less and is resistant to the antimicrobial agents due to an effective proton pump mechanism which maintains optimal cytoplasmic pH levels (11). Enterococci has the natural ability to acquire, gather and has ability for encoding virulence traits of extra chromosomal elements paving way to colonize, it also compete with other bacteria, and resist host defence mecha- nisms leading to pathological changes. These changes may be initiated on production of toxins or indirectly through induction of inflammation $(14,15)$. The pioneering studies by Sundqvist (16) and later by Love et al. (17) demonstrated that, in addition to enterococci, streptococci, lactobacilli, and Actinomyces, obligately anaerobic species of Fusobacterium,Eubacterium spp., Propionibacterium spp., Bifidobacterium spp., Peptostreptococcus micros, and Veillonella species dominated the root canal microflora. Facultative anaerobes belong to the viridans group Streptococci and are commonly implicated in dental abscess. The viridans group Streptococci includes the mutans group (18).

Brook et al. found that the presence of Prevotella, Porphyromonas, Fusobacterium and Peptostreptococcus in pulpitis and dentoalveolar abscess (19). This is in accordance to our study in which few colonies of Porphyromonas and Preptostreptococcus were seen.

It is widely known that the plant oils possess certain medicinal properties and studies have been actively conducted to prove its antimicrobial efficiency. Therefore in the present study antimicrobial effect of zinc oxide with tea tree oil, zinc oxide with cinnamon oil, zinc oxide with peppermint oil and zinc oxide with coconut oil and zinc oxide with eugenol was 
evaluated against Enterococcus faecalis which was the predominant organisms detected in our study .

The components of plant oils are known to interfere with the enzymatic activity, thereby destroying bacterial leading to antimicrobial action. They also block clusters of Gram-positive bacteria, slow down bacterial replication, and remove endotoxin from Gram-negative bacteria to reduce oral pathogenic diseases (20).

In the present study, Agar diffusion method was used. It is one of the most commonly employed technique for evaluation of antimicrobial activity (21). The results show that zinc oxide with tree tea oil has strong inhibitory properties as compared to routinely used zinc oxide eugenol. Our results which is agreement to the findings of the study by Thosar et al. in 2018 (22)' which shows that the tree tea oil with zinc oxide has a better antimicrobial action when compared with zinc oxide eugenol. Another study done by Thosar et al. (23) on five essential oils showed that tree tea oil has significant inhibitory effect on Enterococcus faecalis.

Various studies show the antimicrobial effect of tree tea oil. Ghayathri et al. (24) and Stoica et al. (25) have also shown significant inhibitory effect of tea tree oil on Enterococcus faecalis. The antibacterial effect of tea tree oil is due to terpinen-4-ol, $\alpha$ - terpinol and 1,8-cineole. Terpinen-4-ol enters the cell membrane of microorganisms and acts against its structural permeability. This inturn affects the metabolism of certain microorganisms leading to bactericidal and fungicidal effect (7).

Our study showed significant zone of inhibition by zinc oxide with coconut oil against Enterococcus faecalis .There are no studies in literature about the antimicrobial effect of zinc oxide with coconut oil .The antibacterial property of coconut oil is because of the presence of medium chain fatty acids (26). Devan et al. (27) have shown in 2019 that medium chain fatty acids when used as an root canal irrigant has a significant action against Enterococcus faecalis. Studies show that lauric acid which is constituent of coconut oil exhibits significant antimicrobial activity against $E$. faecalis (28). The inhibitory action of coconut oil may be due to their surfactant activity and their ability to cause cellular lysis by disrupting cell membranes by specific interaction with sites within the microorganism or nonspecific interaction inhibiting physiological function (29).

Zinc oxide with pepper mint oil also showed significant antibacterial action. Thosar et al. (23) in a study against Enterococcus faecalis found that peppermint can act as an effective intracanal antiseptic solution against oral pathogens. Manoj et al. found that peppermint oil has significant action against Enterococcus faecalis (31). Peppermint oil is obtained from the stem, leaves, and flowers of Mentha piperita plant by steam distillation method. Its principal constituents include monoterpinic alcohols mainly menthol, ketones mainly menthones, some monoterpens and oxides. Other active constituents are menthol, menthone, cineol, and several other volatile oils (32).

In the present study zinc oxide with cinnamon oil has shown to have antimicrobial property against Enterococcus faecalis. Panchal et al. (33) compared the antibacterial efficacy of cinnamon extract and calcium hydroxide as intracanal medicament against Enterococcus faecalis and found that cinnamon extract showed good antimicrobial efficacy. The antibacterial activity of cinnamon leaf extract against Enterococcus faecalis is shown to have inhibition zones at $20 \%$ concentration. Cinnamaldehyde, the most abundant component of cinnamon oil is a phenylpropanoid that has proven activity against microorganisms $(34,35)$.

\section{Limitations of the study}

Sample size for the study was fewer for species identification. The use of advanced technique such as polymerised chain reaction, DNA-DNA hybridisation etc would have given more specificity and sensitivity to the species identification. Many other factors such as saliva, mucus layer, clearance capacity, blood flow and normal flora could have had an affect on the results of this study. The antimicrobial effect of the materials was done in vitro in laboratory conditions where intra oral conditions are dynamic with lot of factors involved in antimicrobial action. Thus there is a need for more invivo studies to standardize the microbial activity. Further research is also required to elucidate the mechanistic details as well as to evaluate the toxicity and clinical efficacy. 


\section{CONCLUSIONS}

In this study we found that gram positive facultative anaerobes as the most predominant species. Enterococcus faecalis was found to be the most predominant followed by Streptococcus mutans, peptostreptococcus, porphyromonas gingivalis and actinomyces species. All zinc oxide with oil mixtures has shown antimicrobial activity against Enterococcus faecalis predominance. Hence, this study concludes that apart from traditional use of eugenol, oils such as peppermint oil, tea tree oil, coconut oil and cinnamon oil with zinc oxide can be considered as root canal obturating materials in primary tooth due to their antimicrobial properties.

\section{Conflict of interest: none declared Financial support: none declared}

\section{REFERENCES}

1. Kilian M, Chapple ILC, Hannig M, et al. The oral microbiome - an update for oral healthcare professionals. Br Dent J. 2016;221(10):657-666.

2. Jhajharia K, Parolia A, Shetty KV, Mehta LK. Biofilm in endodontics: A review. J Int Soc Prev Community Dent. 2015;5(1):1-12.

3. Silva LAB, Nelson-Filho P, Faria G, Souza-Gugelmin MCM de, Ito IY. Bacterial profile in primary teeth with necrotic pulp and periapical lesions. Braz Dent J. 2006;17(2):144-148.

4. Narayanan LL, Vaishnavi C. Endodontic microbiology. J Conserv Dent. 2010;13(4):233-239.

5. Doneria D, Thakur S, Singhal P, Chauhan D. Comparative evaluation of clinical and radiological success of zinc oxideozonated oil, modified 3mix-mp antibiotic paste, and vitapex as treatment options in primary molars requiring pulpectomy: An in vivo study. J Indian Soc Pedod Prev Dent. 2017;35(4):346.

6. Rewal N, Thakur A, Sachdev V, Mahajan N. Comparison of Endoflas and Zinc oxide Eugenol as root canal filling materials in primary dentition. J Indian Soc Pedod Prev Dent. 2014;32(4):317.

7. Swamy MK, Akhtar MS, Sinniah UR. Antimicrobial Properties of Plant Essential Oils against Human Pathogens and Their Mode of Action: An Updated Review. Evid Based Complement Alternat Med. 2016;2016:3012462.

8. Chouhan S, Sharma K, Guleria S. Antimicrobial Activity of Some Essential Oils-Present Status and Future Perspectives. Medicines (Basel). 2017;4(3):58.

9. Grossman LI, Oliet S, Del Rio CE. Microbiology. In: Endodontic Practice. Grossman LI, Oliet S, Del Río CE (Editors). 11th ed. Philadelphia: Lea \& Febiger; 1988:234-241.

10. Kutllovci T, lljovska S, Begzati A, et al. Bacteriological identification of selected pathogens in infected primary and young permanent teeth associated with clinical symptoms. OJMM. 2015;05(02):59-68.

11. Alghamdi F, Shakir M. The Influence of Enterococcus faecalis as a Dental Root Canal Pathogen on Endodontic Treatment: $A$ Systematic Review. Cureus. 2020 Mar 13;12(3):e7257.

12. Pinheiro ET, Gomes BP, Ferraz CC, Sousa EL, Teixeira FB, Souza-Filho FJ. Microorganisms from canals of root-filled teeth with periapical lesions. Int Endod J. 2003 Jan;36(1):1-11.

13. Ozbek SM, Ozbek A, Erdorgan AS. Analysis of Enterococcus faecalis in samples from Turkish patients with primary endodontic infections and failed endodontic treatment by real-time PCR SYBR green method. J Appl Oral Sci. 2009;17(5):370-374.

14. Fiore E, Van Tyne D, Gilmore MS. Pathogenicity of enterococci. Microbiology Spectrum. 2019;7(4).

15. Alghamdi F, Shakir M. The Influence of Enterococcus faecalis as a Dental Root Canal Pathogen on Endodontic Treatment: A Systematic Review. Cureus. 2020;12(3):e7257.

16. Sundqvist G. Taxonomy, ecology, and pathogenicity of the root canal flora. Oral Surg Oral Med Oral Pathol. 1994 Oct;78(4):522-30.

17. Love RM. Invasion of dentinal tubules by root canal bacteria. Endodontic Topics. 2004;9(1):52-65.
18. Fowell C, Igbokwe B, MacBean A. The clinical relevance of microbiology specimens in orofacial abscesses of dental origin. Ann $R$ Coll Surg Engl. 2012;94:490-2.

19. Brook I. Microbiology and management of endodontic infections in children. J Clin Pediatr Dent. 2003 Fall;28(1):13-7.

20. Nazzaro F, Fratianni F, De Martino L, Coppola R, De Feo V. Effect ofessential oils on pathogenic bacteria. Pharmaceuticals. 2013 Dec;6(12):1451-74.

21. Balouiri M, Sadiki M, Ibnsouda SK. Methods for in vitro evaluating antimicrobial activity: A review. J Pharm Anal. 2016;6(2):71-79.

22. Thosar NR, Chandak M, Bhat M, Basak S. Evaluation of Antimicrobial Activity of Two Endodontic Sealers: Zinc Oxide with Thyme Oil and Zinc Oxide Eugenol against Root Canal Microorganisms - An in vitro Study. Int J Clin Pediatr Dent. 2018 Mar-Apr;11(2):79-82.

23. Thosar N, Basak S, Bahadure RN, Rajurkar M. Antimicrobial efficacy of five essential oils against oral pathogens: An in vitro study. Eur J Dent. 2013 Sep;7(Suppl 1):S071-S077.

24. Ghayathri VK, Delphine PA, Muralidharan NP. Comparison of antimicrobial activity of tea tree oil extract and hydrogen peroxide with $3 \%$ sodium hypochlorite against Enterococcus faecalis, Candida albicans - An in vitro study. Drug Invention Today. 2020;13(5);744-48.

25. Stoica PE, Chifiriuc MC, Rapa M, Bleotu C, Lungu L, Vlad G, Grigore R, Bertesteanu S, Stavropoulou E, Lazar V. Fabrication, characterization and bioevaluation of novel antimicrobial composites Biotechnol Lett. 2015 May 1;20:10521-35.

26. Peedikayil FC, Remy V, John S, Chandru TP, Sreenivasan P, Bijapur GA. Comparison of antibacterial efficacy of coconut oil and chlorhexidine on Streptococcus mutans: An in vivo study. J Int Soc Prev Community Dent. 2016 Sep-Oct;6(5):447-52.

27. Devan K, Peedikayil F, Chandru T, Kottayi S, Dhanesh N, Suresh Kr. Antimicrobial efficacy of medium chain fatty acids as root canal irrigants: An in vitro study. J Indian Soc Pedod Prev Dent. 2019;37(3):258.

28. Devan K, Peedikayil FC, Chandru TP, Kottayi S, Dhanesh N, Suresh KR. Antimicrobial efficacy of medium-chain fatty acids, $2 \%$ chlorhexidine, and 5\% sodium hypochlorite against Enterococcus faecalis: An in vitro study. Indian J Oral Health Res. 2018;4:47-51.

29. Peedikayil FC. Is coconut oil good for oral health? A review. J Health Res Rev. 2019;6:1-4.

30. Benbelaïd F, Khadir A, Abdoune MA, Bendahou M, Muselli A, Costa J. Antimicrobial activity of some essential oils against oral multidrug-resistant Enterococcus faecalis in both planktonic and biofilm state. Asian Pac J Trop Biomed. 2014;4(6):463-472.

31. Manoj KR, Gopinath P. Antibacterial activity of peppermint oil against isolates of enterococcus faecalis from dental caries. International Journal of Current Advanced Research. 2017 May 28;6(5):3595-96.

32. Ratan R. Handbook of Aromatherapy. A Complete Guide to Essential and Carrier Oils, Their Application and Therapeutic Use for based onpolycaprolactone, chitosan and essential oils. Rom 
Holistic Health and Wellbeing. 2nd ed. Institute of Holistic Health Sciences, Mumbai; 2006.

33. Panchal V, Gurunathan D, Thangavelu L. Comparison of antibacterial efficacy of cinnamon extract and calcium hydroxide as intracanal medicament against $E$. fecalis: An in vitro study. Pharmacogn J. 2018;10(6):1165-8.

34. Gupta A, Duhan J, Tewari S, Sangwan P, Yadav A, Singh G, Juneja $\mathrm{R}$, Saini H. Comparative evaluation of antimicrobial efficacy of
Syzygium aromaticum, Ocimum sanctum and Cinnamomum zeylanicum plant extracts against Enterococcus faecalis: A preliminary study. Int Endod J. 2013;46:775-783.

35. Firmino DF, Cavalcante TTA, Gomes GA, et al. Antibacterial and antibiofilm activities of cinnamomum sp. Essential oil and cinnamaldehyde: antimicrobial activities. The Scientific World Journal. 2018;2018:1-9. 